DR. M. H. HLetcher, Cincinnati, said that a large part of his professional time is devoted to the treatment of these cases. He agreed that this trouble is of local origin, with systematic complications of many varieties, and he considers it interest. ing to note that the trouble in these cases will disappear by local treatment. It is a well-known fact, he said, that the conjunctiva, or other mucous membranes of the body, are irritated in the dry stage of inflammation. Every tissue of the body is subject to irritation, with accompaniments of systemic trouble. Dr. Fletcher believes in a local cause for many disorders. It is a fact, he said, that every disease has its nervous phase; many diseases are now known to be purely nervous which were formerly thought to be organic. Any condition which produces a continual irritation may produce systemic disorders of a severe nature, and he is convinced that the systemic disorders referred to are brought about by the local irritation, which is continuous. There is an effort to throw off this foreign body, and this continuous effort of Nature to restore herself to normal results in the destruction of the tissue about the teeth, with the result that finally a pathologic or diseased habit is established; and in no instance can it be cited that this trouble does not disappear by removal of the teeth Dr. Fletcher said that it has fallen to his lot to do considerable work in the antrum, and in hundreds of examinations of the mouth and skull he has failed to find the antrum as far for ward as the canine, and in but twelve cases in a thousand antrums has he found that the roots of the teeth produced a tubercle in the floor of the antrum, as shown by Gray and other anatomists. He has found abscesses of the molar teeth discharging in the antrum where the roots come against the floor of the antrum; the roots are often uncovered, so far as bone is concerned, just as all practitioners know; on the outer surface of the alveolar process the bone is completely gone and the root covered with nothing but soft tissue. Now it is easy to understand that in gingivitis going up the root in a case in which there is no body covering to the root in the floor of the antrum, there will be extension into the antrum, with all the accompaniments. This disease is something to which Dr. Fletcher thinks stomatologists must give more attention. He has never seen a patient in whom there was not more or less calcareous deposit. He has seen a case of pyorrhea in a child of ten. In other ways the child was perfectly healthy; the disease evidently had been caused by calcareous deposit.

Dr. G. V. I. Brown, Milwaukee, said that it might be of in terest to recall something of the progress of the science of this particular affection, as Dr. Talbot has brought it out. His earlier papers demonstrated, for the first time by actual microscopic study, that this disease was considerably in advance of what he had been accustomed to recognize as diseased tissue of this character. His first series of slides showed that. He next took up the question of autointoxication, and showed by slides the relation of those conditions in this disease, and again of that kind of poison to mineral and other poisons, bringing it all down to the simple proposition of an absorption and taking into the system of poison which was disastrous to tissue, which affects, first of all, that portion supplied by the very small vessels and structures of a transitory nature, such as the alveolar process. Dr. Brown said that we have all heard and read for years of the uric acid diathesis, and its relation to this disease; and now we find that uric acid, while it is a factor and sometimes represents associated disease, it is only one of many disturbing elements of a somewhat similar character.

- Dr. M. H. Fuetcher, Cincinnati, said that this discussion has shown unquestionably the local features of this disease, and according to his mind the proposition is quite simple; with the system loaded with intoxication there is less resistance to any kind of an irritation. If, under these circumstances, there is local irritation in this region, there is as a result a condition which we recognize in the disease. It is a well-known fact, he said, that affections of the kidneys, or other organs, weaken their powers of function, especially that of elimination, laying all the tissues especially liable to disturbance from any kind of irritation. Now, if we supply the irritation about the teeth, we produce the disease. If we remove the irritation and the intoxication, to that extent we relieve the condition and the disease.
Dr. VIDA A. Latitam, Chicago, said that this exhibition of Dr. Rhein's proves almost conclusively that the stand taken by some of the members of the Section, that dentists are pure mechanics, is certainly out of order. Dr. Latham said that we can not do without the scientifie branches. Dr. Rhein's exhibition, she said, to-day has shown that the mechanical filling of those canals, their anatomic bearingrs, the relations of those tissues and the far-reaching effects mentioned by Dr. Talbot, have brought together both the scientific and the mechanical aspects, as perhaps has never before been done. Dr. Latham said that we have to deal with another set of tissues, the mucous glands, which form around and in the peridental membrane, and that there is a bearing of the mucous glands in the peridental tissue which has to deal with the question; these glands are also affected by the end organs, and by the arterial changes. What the exact histologic and anatomic conditions of those mucous glands are we do not know. Dr. Latham declared that it can truthfully be said that in some lines dentistry has advanced far ahead of medicine: But in these particular lines-the scientific and anatomic -we are not advancing as rapidly as in the mechanical.

\section{PALLIATIVES FOR HAY FEVER.}

\section{SOLOMON SOLIS-COHEN, M.D. \\ professor of Clinical Medicine in Jefferson Medical College. PHILADELPIIIA.}

Since 1898 , when I made the first announcement of the value of preparations of the suprarenal gland in the treatment of hay fever, ${ }^{1}$ I have made many observations on myself and others with a view to determine the best of these preparations and the best method of application and administration. I have also made observations with other promising palliatives, especially pollantin. The results may be summed briefly.

\section{SUPRARENAL PREPARATIONS SYSTEMICALIY.}

For some individuals, and in certain seasons, the systemic action of suprarenal preparations suffices to induce and preserve comparative comfort. The stomach, however, as I have previously pointed out, is not an eligible channel of administration. The medicinal substance must be absorbed from some other mucous membrane, where it can escape exposure to the digestive juices and the action of the liver. The eye, the tongue, the nose, the pharynx may be utilized. It was, and still is, possible to attain results by using the old crude preparations of suprarenal substance, but the active principles are more easily managed on account of their effectiveness in the minutest quantities. I have employed both the adrenalin chlorid solution of Takamine and the epinephrin of Abel; the latter being marketed by a manufacturing firm under the name of suprarenalin. Suprarenalin, which appears in the form of a non-hygroscopic powder, may be made into very small tablet triturates, each containing from $1 / 40$ to $1 / 10$ of a grain (1.5 to 6 milligrams) of the active substance, with a small quantity of sugar of milk; and this being placed on the tongue quickly dissolves, is absorbed and becomes therapeutically active. The quantity of sugar of milk used should be the least possible to make a triturate that can be handled. I have to thank Mr. F. E. Morgan, of Philadelphia, for his skill in making for me these very small tablets, as also for his efficient co-operation in my experiments with various diluent powders, as set forth later in discussing the topical action of suprarenal preparations. The dose of suprarenalin that I find effective in my own case is $1 / 20$ grain $(0.003 \mathrm{gm}$.$) , placed on the$

1. Philadelphia Medical Journal, Aug. 13, 1898. 
tongue. This dose may be repeated in from 10 minutes to two hours, according to effect and necessity. When one remains quietly at home in a cool room, in darkness or semi-darkness, one or two doses daily will suffice. If one goes abroad in the city and takes the precaution of wearing dark glasses, a tablet every hour or two may be necessary. If one goes into the country and especially if one has to take a long, hot and dusty railway journey, local measures are likely to become necessary, in addition to the more frequent use of the lingual administration of suprarenalin. The dose varies with different individuals and with the same individual in different seasons, on different days of the same season, and even at different periods of the same day, as position of sun, temperature, humidity, precipitation, dustiness, direction of prevailing wind, cloudiness and other environmental conditions change. As a rule, those who have not before been treated in this manner respond readily to doses as small as $1 / 40$ or $1 / 50$ of a grain of suprarenalin, while those who have used this agent in previous years seem to need larger doses, even, at times, up to $1 / 10$ of a grain.

When suprarenalin is not available or a druggist can not be found to make it into very small tablet triturates, or into small powders, 1 or 2 grains of powdered suprarenal substance may be taken on the tongue and allowed to remain there as long as convenient. There is no objection to swallowing it, but so much as is swallowed. becomes inert, in so far as control of the hay fever syndrome is concerned. Better than this is the use of a compressed tablet ( 3 or 5 grains) of suprarenal substance, allowing it to dissolve in the mouth somewhat after the manner of the ordinary lozenge. But these expedients are crude and ineffective in comparison with the use of the active principle.

Adrenalin chlorid solution is not eligible for use by lingual administration, as in order to obtain the necessary quantity of active principle one would have to hold in the mouth half-a-dram or more of the commercial solution $(1 / 1,000)$, and even if the mouth did not fill additionally with saliva it is practically impossible to continue so holding the fluid long enough for absorption; it must either be ejected or swallowed. Solutions of active principle (either adrenalin chlorid or suprarenalin) may be used by instillation into the conjunctival sac or into the nasal passages; the quantity, however, that may be employed in this way being rarely sufficient for systemic influence, despite its excellent topical effect.

II. SUPRARENAIIN PREPARATIONS, TOPICALLY.

In the majority of cases the systemic administration of suprarenal preparations must from time to time be supplemented by their topical use, while in some cases the topical use alone suffices to keep the patient comfortable, except when the environment is particularly unfavorable. Fresh filtered solutions, powders and ointments of desiccated suprarenal substance, as used by Bates and others and by me, before the active principles had been introduced, are effective, but they are not so easily managed as preparations of adrenalin or of suprarenalin. The last-named substance may be used in solid form by sniffing or insufflation, if sufficiently diluted $(1 / 500$ or $1 / 1,000)$ with an unirritating powder. Both forms of the active principle may be obtained in watery or oily solution (from $1 / 1,000$ to $1 / 4,000$ ) for use by nebula or spray; or rubbed up either with a simple base (petrolatum, lanolin, lard, 1/1,000), or with the addition of a small quantity of a sedative and astringent agent. such as zinc oxid. may he employed as an oint- ment. Before these topical applications are made the nose should be thoroughly cleansed with an alkaline detergent spray, or if the application is to be made to the eye the conjunctiva should be bathed with a sedative collyrium. If ointment or oily spray is to be used, the cleansing spray applied to the nasal passages may also be oily, but if the powder or a watery solution is to be used an oily spray is evidently contraindicated. However, it is always well, some ten or fifteen minutes after the adrenalin application, to use a puff or two of some bland aromatic oily spray, that may act as a protective covering to the mucous membrane. I have given up the use of aqueous solutions of the suprarenal principles, except in the eye for local palliation, and even here in some cases it is better to apply an ointment to the lids. Sometimes I use in the nose an ointment or suppository of suprarenal substance $(1 / 50$ or $1 / 100)$, or, preferably, of adrenalin or suprarenalin $(1 / 1,000)$ in cocoa butter. or a smooth base of lanolin ( 1 part) and benzoinated lard ( 2 parts), with or without the addition of zinc oxid ( 10 to 33 per cent.) or yellow mercuric oxid (1/2 to 2 per cent.). The following formulas are typical :
1. Suprarenalin $\ldots \ldots \ldots \ldots \ldots \ldots \ldots$ gr. $1 / 5$
Wool fat
Purified petrolatum
Benzoinated lard, of each.............
Yellow mercuric oxid ............... $\mathrm{i}$
Mix. Rub well. Dispense in tin tube.
Sign: For application to eye-lids or nasal passages.
2. Suprarenalin $\ldots \ldots \ldots \ldots \ldots \ldots \ldots \ldots$ gr. $1 / 5$
Wool fat ..............................
Ointment of zinc oxid (benzoinated) .....3ii
Mix. Rub well. Add a few drops of olive oil if needed to make a smooth ointment. Dispense in a tin tube. Sign: For application to nasal passages.

More frequently, however, $I$ find it advisable to use a snuff made according to some such formula as follows:

3. Suprarenalin $\ldots \ldots \ldots \ldots \ldots \ldots \ldots \ldots$ l part Zinc stearate (comp.) ................

Heavy magnesium earbonate .......900 parts Mix. Triturate well.

4. Suprarenalin $\ldots \ldots \ldots \ldots \ldots \ldots \ldots \ldots, 1$ part Zine oxid $\ldots \ldots \ldots \ldots \ldots \ldots \ldots \ldots \ldots 100$ parts

Bismuth subcarbonate $\ldots \ldots \ldots \ldots \ldots \ldots+\ldots 00$ parts Mix. Triturate well.

5. Suprarenal gland substance $\ldots \ldots \ldots \ldots$ l part Zinc stearate $\ldots \ldots \ldots \ldots \ldots \ldots \ldots, 20$ parts Zine oxid .................. 80 parts Mix. Triturate well.

The one I use most frequently is:

6. Suprarenalin ................ l part Bismuth subcarbonate $\ldots \ldots \ldots \ldots \ldots \ldots .300$ parts Zinc oxid ...................... 300 parts Zince stearate (comp) ...........200 parts Mix. Triturate well.

These preparations can be snuffed up into the nasal passages or may be insufflated by means of a powderblower. I have used as diluents of the snuff a number of other agents, as, for example, boric acid, sodium borate, sodium bicarbonate, starch powder. I have also added at times to snuff powders or as constituents of ointments or "nasoids" ("nasal suppository") minute quantities of menthol, camphor, cocain, antiyprin, acetanilid. With some individuals boric acid or sodium borate or sodium bicarbonate answers better than the diluents given in the foregoing formulæ, but in most cases they irritate more or less. Starch powder-marante being the form used-proved irritating in every case in which it was tried, so that but a few experiments, some half dozen, were made with it. Menthol, camphor, antipyrin and acetanilid proved decidedly irritating in my own case. hut for a few patients were found to be 
desirable additions, assisting in the cleansing and opening of the nasal passages and giving an agreeable aftersensation. Cocain and alypin were always palliative, the former assisting markedly to free the respiration, yet for prudential reasons I rarely prescribe it. Orthoform proved too irritating to be persisted with. Despite the initial smarting it produces, anesthesin (1 to 500) was useful in a number of cases. But for its lightness, zinc stearate would have been by far the best diluent powder of any that was experimented with. Being so light, unless great care is observed in snuffing it up or in insufflating it, it is quickly carried into the pharynx and sometimes into the larynx, inducing a rather disagreeable choking sensation, which, however, soon passes off. When the patient can be trusted to make the application carefully, a snuff of 1 part of suprarenalin in from 100 to 1,000 parts of zinc stearate meets all the requirements. The bismuth subcarbonate, the heavy magnesium carbonate and the zinc oxid were added as unirritating substances, possessing sufficient weight to be available. In addition, zinc oxid has a slight astringent effect, bismuth subcarbonate is slightly sedative and both act as protective coverings. Perhaps the best diluent of all, could it be obtained, would be a serum powder prepared from the blood of healthy animals-preferably horses or goats, to avoid the danger of unrecognized tuberculosis. The anti-tetanic serum powder and antidiphtheritic serum powder on the market would answer, but these are unnecessarily expensive, their specific antitoxic properties not being requisite for this special use. I have also used with success a snuff consisting of suprarenal gland substance ( 1 to 5 per cent.) zinc stearate and bismuth subcarbonate or zinc oxid. This is less expensive than the suprarenalin snuffs.

\section{IIr. POLLANTIN}

Dunbar's antipollenic serum is a distinct addition to our resources and its effects are positive in from 30 to 50 per cent. of the cases met with. I have not found it to be more than a palliative, however, in any instance, and in those cases in which the suprarenalin preparations are effective pollantin offers no advantage over them that I have been able to see or to experience. In not a few cases pollantin or suprarenal snuff can be used indifferently with about the same useful palliative result. This has been my own personal experience. Either pollantin (spring) or suprarenalin with zine and bismuth powder gives me relief for from two to ten hours, according to environmental conditions; both failing to give relief for more than ten or fifteen minutes, while on a long and dusty railway journey. In some cases, however, in which the suprarenalin preparations fail entirely, pollantin controls the symptoms. In a less number of cases pollantin fails and suprarenalin succeeds. In still other cases, about one-third of all, according to my observation, both pollantin and suprarenalin fail to have any marked effect.

Both pollantin and suprarenalin snuffs must be used properly to be effective. The nose should first be cleansed, then a small quantity should be sniffed into each nostril and thoroughly distributed by titillation after the manner of the elegant snuff-takers of former generations. ${ }^{2}$ This titillation should be done with the head thrown well back, so as to bring the nasal fossæ as near as possible into a vertical position. For this purpose one may lie down and should, in any event, remain

2. I owe these suggestions to one who, although a layman in medicine, is an "adept" in hay-fever "treatments," as well as in furisprudence. recumbent for some ten minutes after the snuff-taking, if at all possible. The snuffing of pollantin or the snuffing or insufflation of suprarenalin powder, as the case may be, should be done the first thing in the morning, preceding the toilet, and if thoroughly done and if the ten or fifteen minutes of recumbency be observed, such an application will protect for several hours; if not for the whole day, under favorable conditions; especially if the additional precaution of using two or three puffs of oily spray as a protective be carried out, as advised in a previous paragraph. In the use of pollantin one should be careful to prescribe and to obtain the "spring pollantin" in the spring and the "autumn pollantin" in the fall. As I have previously suggested, ${ }^{3}$ it would be still more desirable that a number of specific pollantins, as grass, rose, goldenrod, ragweed, etc., should be prepared, in which case the number of persons relieved by one or the other of these preparations would doubtless be much greater than the 30 to 50 per cent. of all sufferers for whom the preparations now in the markot are suitable and effective.

\section{INTERNAL MEDICATION.}

In some instances in which one or more of the measures already described proves but partially successful in controlling the symptoms of hay fever, relief may be aided and often completely achieved by the judicious internal administration of belladonna or its active principle or some modification thereof. Minute doses are necessary, and these should be taken at intervals determined experimentally in the individual case. I find it convenient to use atropin sulphate or hyoscin hydrobromate or, sometimes, eumydrin, which last bears the same relation to atropin that heroin bears to morphin, being a methyl substitution product (methyl-atropin-nitrate). 'The dose of the hyoscin salt or the atropin salt is from $1 / 2,500$ to $1 / 500$ of a grain (1/40 to $1 / 8$ milligram). Ordinarily $I$ employ about $1 / 2,000$ of a grain. The dose of eumydrin is from $1 / 200$ to $1 / 64$ of a grain ( 0.3 to 1 milligram). 'The following are illustrative formulas:

7. Hyoscin hydrobromate ......gr. 1/100 0006 Camphor monobromate ............ gr. Milk sugar .................. sufficient 12 Mix. Triturate well. Divide into 20 tablets (or capsules ).

8. Atropin sulphate $\ldots \ldots \ldots \ldots \ldots$ gr. $1 / 50 \quad 1001$ Camphor monobromate .........gr. v Peruvian balsam .............sufficient Mix. Divide into $20,24,32$ or 40 equal parts and dispense in capsules.

9. Eumydrin $\ldots \ldots \ldots \ldots \ldots \ldots \ldots \ldots \ldots$ gr. i $\quad 105$ Milk sugar .............. sufficient Mix. Triturate well. Divide into 100 or 200 tablets (or capsules).

The dose of any of the foregoing preparations is one tablet (or capsule), to be repeated every ten to ninety minutes until the sneezing and running of the nose are controlled, after which one tablet (or capsule) may be taken every second, third or fourth hour, as may be necessary to maintain the effect. Eumydrin, if given in doses of $1 / 200$ or 1/100 grain, is, of course, not to be repeated so frequently as atropin or hyoscin in doses of $1 / 2,000$ or $1 / 1,000$ grain. Its effects, moreover, are much slower in development.

Patients are told what the unpleasant effect of an overdose of any of these agents would be, and are warned not to take sufficient of the drug to cause notable dilatation of the pupil or dryness of the throat or disturbance of vision. They are also advised to take not less than

3. Srstem of Phrsiologic Therapeutics, vol. xi, page 64 
a wineglassful of water with each dose. 'The addition of camphor mouobromate seems useful, and sometimes a small quantity of quinin, say $1 / 8$ to $1 / 2$ of a grain, may also be added to each dose, although this has not the same applicability in hay fever as in ordinary coryzas.

In many cases it will also be found useful to administer the so-called antilithic remedies, as piperazin quinate, lithium citrate and sodium salicylate. In a few instances i have found a combination of sodium salicylate and sodjum benzoate beneficial.

The following formulas are illustrative:

10. Piperazin quinate

Lithium citrate

Sodium salicylate (true) of each. gr. ii 20

Mix. For one capsule. Sign: One capsule with half a tumblerful of water, after meals and at bedtime (4 times a day).

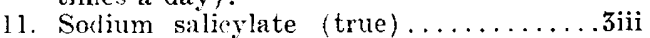

Sodium benzoate (true) ............

Essence of pepsin............f fii

Tincture of nux vomica..........f3i

12

24
60

Peppermint water, sufficient to make...f 3 iv 120

Sign: 2 fluidrams (teaspoonfuls) in (or with) onehalf tumblerful of water, every fourth hour, for four (or five) doses daily.

Mild alkaline carbonated mineral waters are always helpful and patients should be advised to take freely during the hay fever season any palatable water of these groups; for example, one of the numerous American lithia or "Vichy" waters or imported Vichy (Celestin), or Giesshuebl or Fachingen. If preferred, tablets of lithium carbonate or lithium citrate may be used to make an artificial mineral water.

\section{ASTHMATIC PAROXYSMS.}

If the palliative measures already described and the general precautions to be set forth in conclusion are carried out systematically, asthma is not likely to occur. To relieve an attack, a tablet of suprarenalin may be placed on the tongue (1/10 grain, repeated, if necessary, every 10 to 20 minutes) ; or suprarenalin or adrenalin solution $(1: 1000)$ may be applied to the nose by spray or cotton wad; but a hypodermatic injection of 10 to 20 minims of adrenalin or suprarenalin solution (1:1000) is usually the quickest method. If for any reason the suprarenalin preparations are contraindicated, chloroform or ethyl chlorid may be inhaled with due care and not to the induction of general anesthesia. Patients very prone to asthma may be given prophylactic inhalations of ethyl iodid ( 3 to 10 minims every 3 to 4 hours); or one of the following prescriptions may be taken:

12. Aspidospermin hydrochlorate .......gr. iii Hyoscin hydrobromate ........... $1 / 100$

Peruvian balsam ...............sufficient Mix. Make 24 capsules.

Sign: One every hour for 10 to 12 hours daily, or as needed.

13. Morphin hydrochlorate .........gr. 1/6 Strychnin sulphate ............gr. 1/30 Hyoscin hydrobromate .......gr. 1/250 Atropin sulphate ............... 1/250 Sterilized water ............... $x$ Dissolve. Sign: Use by hypodermic injection.

14. Codein sulphate ...............gr. ii 112 Hyoscin hydrobromate ..........gr. $1 / 40$. 1002 Mix. Make 6 (or 8 ) pills. Sign: One pill at 6 or 7
p. m. and one pill at 8 or 9 p. m.; a third pill may be taken at $10 \mathrm{p} . \mathrm{m}$. or later if needed.

15. Strong tincture of passiftora incarnata..f 3 ii 601

Dose. 10 to 30 drops in water every 10,20 or 30 minutes, as needed, to relieve distress in breathing. Four fluidrams must not be exceeded. Should drowsiness, vertigo or nausea occur, the medicine is to be discontinued.

It is only rarely that the last prescriptions will become necessary. Obviously such measures demand care in application and the indications for their use must be clear.

\section{GENERAL PRECAUTIONS.}

Those who are able to go to an immune region usually need no additional palliation. The less fortunate must, in addition to the use of one or more of the expedients detailed in the foregoing paragraphs, observe certain precautions. Exposure to dust, to smoke, to direct sunlight, to heat, must be avoided or minimized. Sometimes it is advisable to wear cotton filters in the nostril when exposed to the dust of city streets, when walking or driving on country roads, and especially during railway trips. Dark glasses will also be found extremely useful. Hurry and excitement are to be avoided, if possible. The bowels, the skin and the kidneys must be kept normally active or even a little stimulated.

Dict must be simple and on the whole sparing. Shellfish and elaborate mixtures are usually harmful. Meat is to be avoided or cut down to the lowest possible point. Pastries and sweets should be avoided. Concerning fruits, marked idiosyncrasies exist. Peaches, if pared, can usually be enjoyed, but the fuzzy skin distresses many persons. Strawberries eause aggravation of symptoms in a few hay-fever patients. Melons are not only palatable and refreshing, but have a slight diuretic action that is often helpful. A tepid bath, followed by a brisk cold rub, just before going to bed, and a hot sponge, followed by a brisk cold rub, in the morning, after the rest following pollantin or suprarenanal snuffing, will be found to conduce very much to comfort. Also advantage should be taken of every opportunity for rest and quiet.

By utilizing some or all of the palliative measures described, not in unintelligent routine, but varying the expedients with the individual and the occasion, the vast majority, probably at least nine-tenths of those hay-fever sufferers who are unable to take the best course-namely, flight to a region of peace-may be enabled to get through their season of tribulation with a minimum of discomfort, and even to enjoy, from time to time, hours and sometimes days of complete relief. In these intervals they have opportunity to realize the truth of the poet's utterance. "Tife's keenest pleasure is surcease of pain."

\section{THE, VALUE OF ROUTINE URINE EXAMINA- TION.}

A STATISTICAL INQUIRY.*

$$
\begin{aligned}
& \text { M. H. FUSSELL, M.D. } \\
& \text { PIILADELPHIA. }
\end{aligned}
$$

If an excuse for writing a paper of the above title is necessary, it may be found in an article published in The Journal of the American Medical Association, March 18-25, 1905, by Dr. Richard C. Cabot, Boston. The title of that paper is: "A Clinical Examination of the Urine. A Critical Study of the Commoner Methods."

The last two paragraphs of the paper are as follows: - The attempt to estimate the anatomic condition of the kidney by the measurement of albumin and the search for casts is fallacious in the extreme." "The most reliable data about the urine are those most simply and quickly obtained, the twenty-four hour quantity, the spe(ific gravity and the color."

To quote the title of the paper and these last para-

* Read by title before the Philadelphta County Medical Societr 